\title{
Fractura osteocondral traumática de rodilla en edad pediátrica. Reporte de un caso
}

\author{
Traumatic osteochondral knee fracture in pediatric age. Report of a case
}

\section{Fratura osteocondral traumática do joelho em idade pediátrica. Relato de um caso}

Martín Sierra ${ }^{1}$, Marcelo Dupont ${ }^{2}$, María Elena Pérez ${ }^{3}$

RESUMEN

Las fracturas osteocondrales (FOC) traumáticas de rodilla en la edad pediátrica, son lesiones que acompañan hasta un 30\% de las luxaciones agudas de rótula (LAR). Si no se mantiene una elevada sospecha clinica, es frecuente su retraso diagnóstico, pudiendo generar potenciales complicaciones. A propósito, presentamos el caso de una paciente de 12 años con una FOC post LAR que pasó inadvertida en la primera consulta, requiriendo la fijación del fragmento osteocondral con tornillos HCS a los 5 meses, logrando un excelente resultado funcional a los 54 meses de seguimiento.

Palabras clave: edad pediátrica, fractura osteocondral, luxación aguda de rótula, rodilla.

\section{ABSTRACT}

Traumatic osteochondral fractures (OCF) of the knee in pediatric age are injuries that accompany up to $30 \%$ of acute patellar dislocations (APD). If high clinical suspicion is not maintained, its diagnostic delay is frequent, and may generate potential complications. Incidentally, we present the case of a 12-year-old patient with a post-APD OCF that went unnoticed in the first consultation, requiring fixation of the osteochondral fragment with HCS screws at 5 months. Achieving an excellent functional result at 54 months follow-up.

Keywords: pediatric age, osteochondral fracture, acute dislocation of the patella, knee.

RESUMO

As fraturas osteocondrais traumáticas (FOC) do joelho em idade pediátrica são lesões que acompanham até 30\% das luxações agudas da patela (LAP). Se uma alta suspeita clínica não for mantida, seu atraso no diagnóstico é frequente e pode gerar complicações potenciais. A propósito, apresentamos o caso de um paciente de 12 anos com FOC pós-LAP que passou despercebido na primeira consulta, exigindo fixação do fragmento osteocondral com parafusos HCS em 5 meses. Obtendo um excelente resultado funcional aos 54 de acompanhamento

Palavras-chave: idade pediátrica, fratura osteocondral, luxação aguda da rótula, joelho.

\section{INTRODUCCIÓN}

La incidencia de luxación aguda de rótula (LAR) es de aproximadamente 43 casos cada 100.000 personasaño. Correspondiendo a entre un 2 y un 3\% de todas las lesiones de la rodilla ${ }^{(1,2,3,4)}$. La mayoría de ellas se produce en la segunda y tercera década de la vida, siendo factores de riesgo conocidos; el sexo femenino, los antecedentes familiares, la displasia de rótula, la patela alta, la hiperlaxitud, así como enfermedades sistémicas que causen esta hiperlaxitud como es el sindrome de Marfan o el sindrome de Down, entre otros $^{(5)}$.

Se estima que de un 30 a 50\% de las LAR generan fracturas osteocondrales $(6,7)$. En especial durante el primer episodio(7). Las radiografias simples (RX) pueden mostrar resultados normales hasta en el $44 \%$ de los $\operatorname{casos}^{(6)}$, por lo que es fundamental mantener una elevada sospecha clinica, a fin de solicitar estudios complementarios como puede ser la resonancia nuclear magnética (RNM) o, eventualmente, recurrir a la artroscopia de rodilla con el fin de llegar a un diagnóstico certero( ${ }^{(8,9,10)}$

Las fracturas osteocondrales se topografian mayoritariamente en la cara articular medial de la rótula, a nivel del cóndilo femoral lateral o en ambos sitios $^{(11)}$. Más del $60 \%$ de las lesiones se vinculan a las actividades deportivas ${ }^{(5)}$.

Dejado a su libre evolución estas lesiones generan derrame articular recidivante, dolor, limitación funcional, incluso mayor daño cartilaginoso vinculado a la agresión que causa el cuerpo libre osteocondral al cartílago articular sano, favoreciendo el desarrollo de artrosis precoz. De lo que se desprende la importancia de realizar el diagnóstico y tratamiento en forma oportuna ${ }^{(12)}$

\section{REPORTE DE CASO}

Se trata de un paciente de sexo femenino de 12 años, estudiante, procedente de Montevideo, sin antecedentes personales patológicos a destacar, deportista amateur. En setiembre del 2016 mientras practicaba gimnasia ritmica sufre un traumatismo indirecto de rodilla izquierda quedando con dolor, edema y limitación funcional a dicho nivel. Consultando en la emergencia del Centro Hospitalario Pereira Rossell (C.H.P.R.), donde se indica analgesia vía oral y reposo deportivo por tres semanas. Luego de la primera consulta la paciente reconsulta en tres oportunidades en emergencia por gonalgia y episodios de derrame articular, donde se indicó reposos deportivo y analgésicos. En junio del 2017 la paciente consulta en policlínica de traumatología del C.H.P.R., destacándose de la anamnesis la presencia de dolor intermitente de

1 Clinica de Traumatologia y Ortopedia Pediátrica, Facultad de Medicina, Universidad de la República, Uruguay. Correo electrónico: msierragarcia@gmail.com

2Asistente de Clinica de Traumatologia y Ortopedia Pediátrica. Correo electrónico: mdup5@icloud.com ORCID: 0000-0001-5936-3601

3Profesor Director de Clinica de Traumatologia y Ortopedia Pediátrica. Correo electrónico: meperezcarrera@gmail.com ORCID: 0000-0001-5778-2267 
tipo mecánico, en especial cuando practicaba actividad deportiva, presentando episodios de derrame articular recurrente, incluso limitando el perimetro de marcha sin dolor a 500 metros, sin nuevos episodios de luxación patelo-femoral. Del examen físico se destaca, discreto derrame articular en rodilla izquierda, dolor leve a la flexo-extensión máxima, aumento de la excursión externa de la rótula en comparación con la contralateral, no signo de la $J$ caracterizado por el encarrilamiento defectuso de la patela durante su discursión en la troclea, rodilla estable, sin signos meniscales, piel sana, sin alteraciones neurovasculares. En la consulta se solicitaron $r x$ en forma urgente de frente y perfil de rodilla (Figura 1), que muestra la presencia de un fragmento osteocondral caracterizado por una imagen de doble contorno a nivel del cóndilo femoral externo. Se completó la valoración imagenológica con una resonancia nuclear magnética (RNM) (Figura 2).
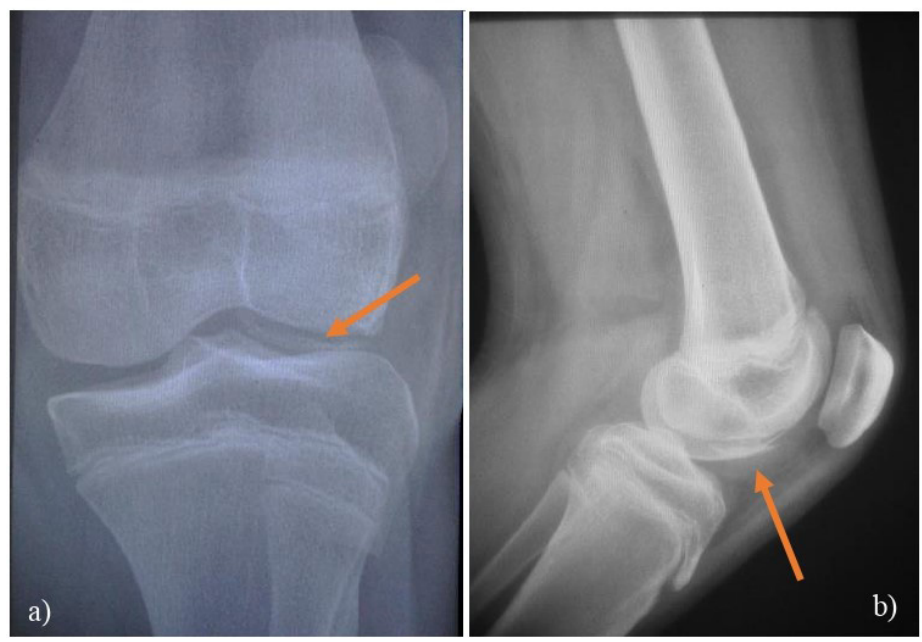

Figura 1. a) Rx de frente de rodilla izquierda de nuestro paciente donde se aprecia un cuerpo libre intrarticular correspondiente a un fragmento osteocondral del cóndilo femoral externo (flecha). b) Rx de perfil de la misma rodilla donde observa una imagen en doble contorno (flecha).

Fuente: elaboración propia.

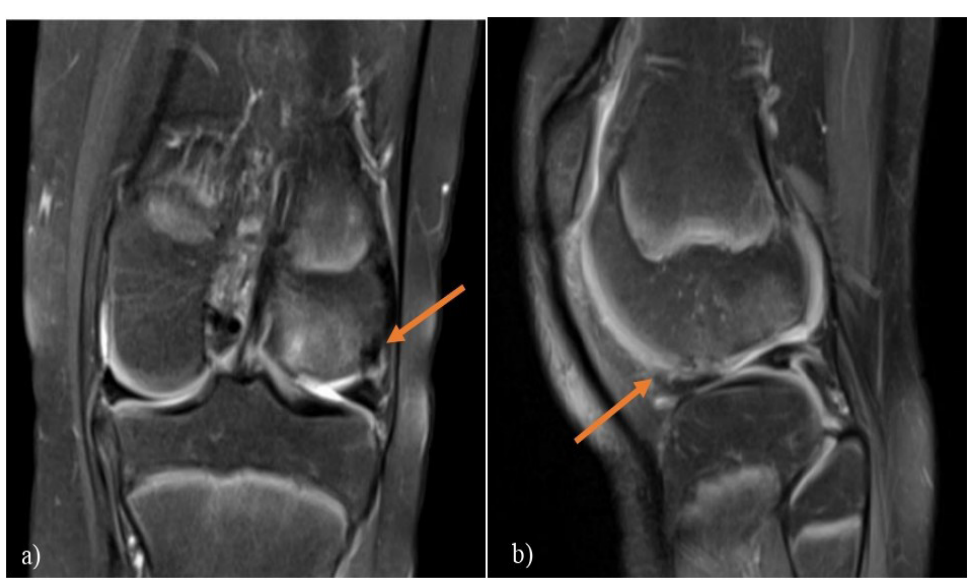

Figura 2. a) RNM T2 de rodilla izquierda, corte coronal, de la paciente donde se aprecia la solución de continuidad a nivel del cóndilo femoral externo de la rodilla izquierda (flecha). b) Corte sagital de la misma rodilla (flecha).

Fuente: elaboración propia.

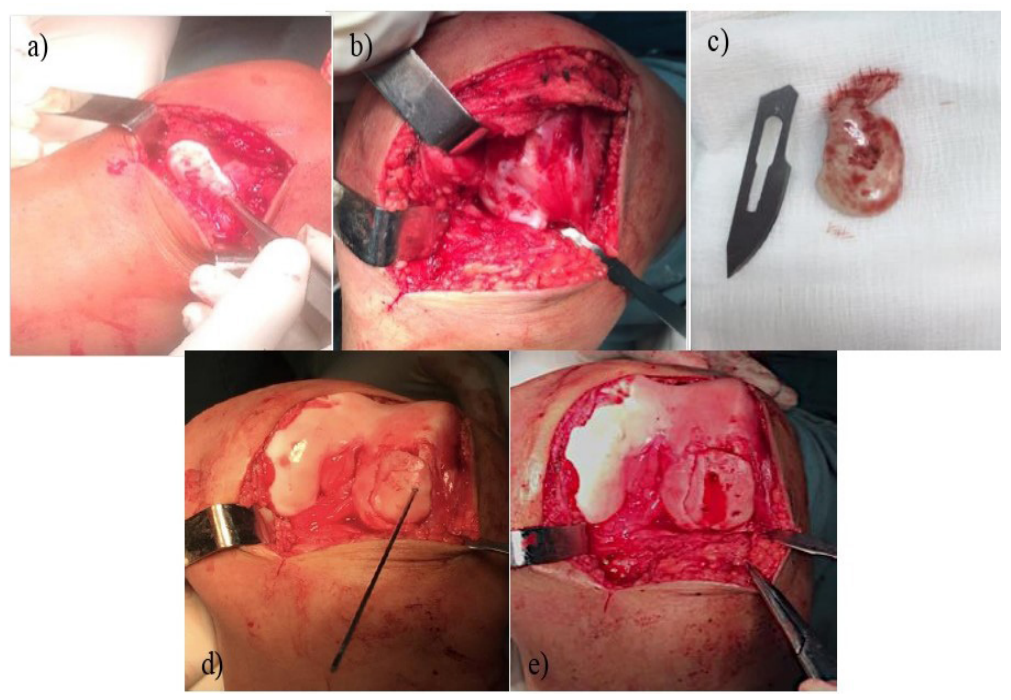

Figura 3. a) Fragmento osteocondral en receso lateral. b) Lecho cruento a nivel del cóndilo femoral lateral. c) Fragmento osteocondral comparado con hoja de bisturi 21. e) Fijación transitoria del fragmento osteocondral. f) Fijación definitiva con tornillos sin cabeza.

Fuente: elaboración propia.

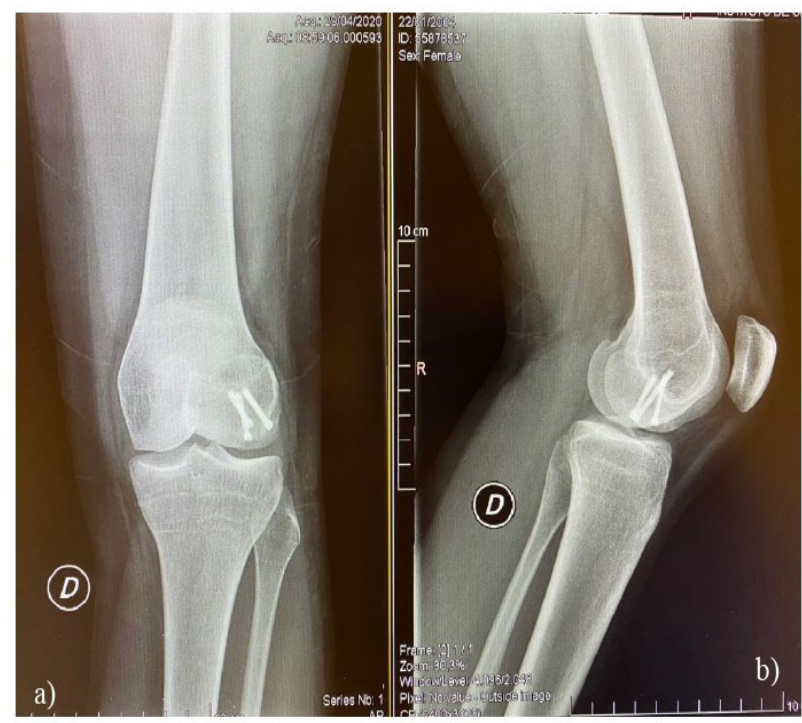

Figura 4. a) Rx frente de rodilla izquierda a 54 meses de la cirugia. b) RX perfil de rodilla izquierda a 54 meses de la cirugía.

Fuente: elaboración propia.

A los 5 meses y 19 días de la lesión inicial se realizó la fijación del fragmento osteocondral mediante un abordaje para patelar lateral, el fragmento osteocondral fue de aproximadamente $4 \mathrm{~cm}$ por $2,5 \mathrm{~cm}$, realizándose la fijación del mismo mediante osteosintesis con 3 tornillos HCS ${ }^{\circledR}$ en su lecho. Las imágenes de la cirugía se muestran en la Figura 3. La paciente cursó un post operatorio inmediato sin complicaciones, no se utilizaron dispositivos de inmovilización, se permitió la deambulación con el uso de muletas. Se comenzó la movilización en forma precoz y la fisioterapia a las 4 semanas. A las 6 semanas comenzó con apoyo parcial progresivo. A las 8 semanas la paciente deambulaba sin asistencia. A los 6 meses de la cirugía la paciente se presentaba asintomática, retomando en forma progresiva la actividad fisica. 
A los 54 meses de follow up la paciente presentaba un International Knee Documentation Committee (IKDC) de 100. Este es un score subjetivo, que evalúa 3 parámetros principales; la sintomatología, los deportes y las actividades de la vida diaria, a través de 18 items que utilizan una escala numérica, lo que genera valores de 0 a 100 puntos. Correspondiendo 100 puntos a una rodilla sin limitación. Este es el caso de nuestra paciente (Figura 4).

\section{DISCUSIÓN}

La población pediátrica es más susceptible a las FOC dado la capa calcificada del cartílago de crecimiento no está completamente formada causando una debilidad entre el cartilago y el hueso subcondral(13). La causa más frecuente de este tipo de lesiones está vinculada a episodios de LAR. Aproximadamente un 30\% de las LAR generan FOC, como plantea Seeley et al. ${ }^{(6)}$ en su trabajo donde sobre 122 consultas por LAR, 46 de ellas presentaban FOC. Chotel et al. ${ }^{(7)}$ estiman que del $30 \mathrm{al}$ $50 \%$ de los episodios de LAR generan FOC, en especial durante el primer episodio, sugiriendo además que según la posición de la rodilla al momento de la luxación se podria inferir la topografia de la lesión. Otros autores como Lee at al ${ }^{(4)}$, Kramer et al. ${ }^{(13)}$ y Felus et al..${ }^{(14)}$, refieren que de un tercio a más de la mitad de las luxaciones de rótula asocian FOC. Lo que marca la importancia de elevar al máximo la sospecha clínica ante un paciente que consulta por un episodio traumático de la rodilla, dado que el mismo puede corresponder a luxación de rótula que se redujo en forma espontánea. Otra causa menos frecuente de este tipo de lesiones se corresponde con los traumatismos indirectos, en general por un mecanismo de cizalla en valgo que provoca el impacto entre el platillo tibial y el cóndilo femoral ${ }^{(13)}$. Tipicamente, esta secuencia traumática causa lesiones en el área de carga del cóndilo femoral lateral, como la que presenta nuestra paciente.

El otro diagnóstico diferencial que siempre debe ser descartado, es la osteocondritis disecante, tipica de pacientes dentro de este grupo etario, pero que en la gran mayoria de los casos se topografía a nivel del sector lateral del cóndilo medial y no va precedido de un evento traumático.

Las rx simples son una herramienta diagnóstica muy valiosa. Es importante contar siempre con radiografias de frente y perfil de rodilla ante un evento traumático. Los enfoques axiales a $30^{\circ} \mathrm{y} / \mathrm{o} 45^{\circ}$, en algunos casos se pueden hacer las incidencias en esos dos ángulos, nos permiten valorar alteraciones fundamentalmente de la articulación femoro patelar. Es de destacar que el componente óseo de la FOC se puede percibir con este tipo de estudio. En el caso de nuestra paciente, probablemente, haber adoptado esta conducta nos hubiera permitido realizar el diagnóstico en forma precoz.

A pesar de la relevancia de las rx, los trabajos de Seelly et al. ${ }^{(6)}$ reflejan que un $44 \%$ de las FOC presentaban rx normales y en el trabajo de Kramer et al. ${ }^{(13)}$ hasta $36 \%$ de las FOC no se lograron diagnosticar con rx. Consideramos, por tanto, importante la realización de una RNM en los pacientes que padecieron una LAR, siendo la misma una herramienta imagenológica muy importante en el diagnóstico de estas lesiones, con un 90 a $95 \%$ de especificidad ${ }^{(13)}$. En el caso de la paciente en cuestión, la RNM fue de utilidad en la planificación quirúrgica permitiéndonos valorar la topografia y la magnitud del fragmento osteocondral, así como también nos permitió descartar lesiones asociadas, que pueden tener implicancias terapéuticas.

La cirugia es la opción más acertada para el tratamiento de las $F O C^{(7,5,13,15)}$. Es preferible realizar la fijación a la remoción de los fragmentos, en especial si los mismos son grandes y pertenecen a la zona de carga. Fragmentos pequeños, menores de $1 \mathrm{~cm} 2$ aproximadamente, pueden ser removidos, sin generar grandes repercusiones funcionales y sin aumentar en forma significativa el riesgo artrogénico(4).

Los implantes utilizados para la fijación del fragmento osteocondral son tema de debate, existiendo múltiples opciones en el mercado. Lo que es claro es que no existe un dispositivo que haya mostrado mejores resultados en la bibliografía revisada. Kramer et. al. en su trabajo comparan la utilización de tornillos de compresión, tornillos sin cabeza e implantes bioabsorbibles y concluyen que no hay evidencia suficiente como para elegir un implante sobre otro(13). Otros materiales como los que utilizó Kumhashi et al. ${ }^{(12)}$ que utilizaron pines de hueso autólogo del paciente, extraídos de la diáfisis tibial, marcan como principales ventajas que este material tiene buena fuerza mecánica, no es necesaria su remoción y a diferencia de los materiales bioabsorbibles no genera alergia. Como desventaja, resalta el riesgo de fractura del sitio donante. Basados en este análisis, los tornillos HCS, como los que utilizamos en nuestra paciente, son una buena opción, con la cual contamos en nuestro medio y estamos habituados a su uso.

En el post operatorio no se utilizaron dispositivos de inmovilización, se permitió la deambulación con el uso de muletas sin cargar peso sobre el miembro operado por 6 semanas, se comenzó la movilización en forma precoz y la fisioterapia a las 4 semanas. A partir de la sexta semana se permitió la carga asistida en forma progresiva. A las 8 semanas la paciente deambulaba sin asistencia. A los 6 meses de la cirugia la paciente se presentaba asintomática, retomando en 
forma progresiva la actividad física. A 54 meses de la cirugia la paciente practica actividad física en forma diaria, cursando el Liceo Militar, sin presentar ninguna sintomatología a nivel de su rodilla.

\section{CONCLUSIONES}

Presentamos un caso de una paciente de 12 años, deportista, con una FOC traumática de rodilla, con 3 años de evolución, producida en el contexto de una $L A R$, que no fue diagnósticada hasta transcurridos 5 meses de la lesión inicial. Destacando la importancia de realizar una RNM frente a este tipo de eventos, en especial en el primer episodio. Se realizó la fijación del fragmeto con tornillos HCS. Se mantuvo en descarga por 6 semanas, a los 6 meses retomó la actividad física. Obteniendo excelentes resultados funcionales, con un alto grado de satisfacción de la paciente, que volvió al ejercico en un nivel igual al previo.

\section{REFERENCIAS}

1. Johnson DS, Turner PG. Management of the first-time lateral patellar dislocation. Knee [Internet]. 2019;26(6):1161-5. Available from: https://doi.org/10.1016/j.knee.2019.10.015

2. Stefancin JJ, Parker RD. First-time traumatic patellar dislocation: A systematic review. Clin Orthop Relat Res. 2007;(455):93-101.

3. Gravesen KS, Kallemose T, Blønd L, Troelsen A, Barfod KW. High incidence of acute and recurrent patellar dislocations: a retrospective nationwide epidemiological study involving 24.154 primary dislocations. Knee Surgery, Sport Traumatol Arthrosc. 2018;26(4):1204-9.

4. Lee BJ, Christino MA, Daniels AH, Hulstyn MJ, Eberson CP. Adolescent patellar osteochondral fracture following patellar dislocation. Knee Surgery, Sport Traumatol Arthrosc. 2013:21(8):1856-61.

5. Seeley M, Bowman KF, Walsh C, Sabb BJ, Vanderhave KL. Magnetic resonance imaging of acute patellar dislocation in children: Patterns of injury and risk factors for recurrence. J Pediatr Orthop. 2012:32(2):145-55.

6. Seeley MA, Knesek M, Vanderhave KL. Osteochondral injury after acute patellar dislocation in children and adolescents. J Pediatr Orthop. 2013:33(5):511-8.

7. Chotel F, Knorr G, Simian E, Dubrana F, Versier G. Knee osteochondral fractures in skeletally immature patients: French multicenter study. Orthop Traumatol Surg Res 2011:97(8 Suppl.):154-9.

8. Enea D, Busilacchi A, Cecconi S, Gigante A. Late-diagnosed large osteochondral fracture of the lateral femoral condyle in an adolescent: A case report. J Pediatr Orthop Part B. 2013;22(4):344-9.

9. Song KS, Min BW, Bae KC, Cho CH, Lee SW. Chondral fracture of the lateral femoral condyle in children with different treatment methods. J Pediatr Orthop Part B. 2015;25(1):43-7.

10. Shaikh AH, Stanclik J, Murphy PGD. A rare case of bilateral nonweight bearing posterior aspect of lateral femoral condyle osteochondral fracture and its management. BMJ Case Rep. 2014:2014:1-3.

11. Bhatt J, Montalban ASC, Wang KH, Lee H Du, Nha KW. Isolated osteochondral fracture of the patella without patellar dislocation. Orthopedics. 2011;34(1):54.

12. Kumahashi N, Kuwata S, Imade S, Kono M, Takuwa H, Uchio Y. Fixation of osteochondral fractures of the patella using autologous bone screws when reconstructing the medial patellofemoral ligament after recurrent patellar dislocation: Report of two cases. J Orthop Sci. 2014:19(2):359-64.

13. Kramer DE, Pace JL. Acute Traumatic and Sports-Related Osteochondral Injury of the Pediatric Knee. Orthop Clin North Am [Internet]. 2012;43(2):227-36. Available from: http://dx.doi. org/10.1016/j.ocl.2012.02.001

14. Felus J, Kowalczyk B, Lejman T. Sonographic evaluation of the injuries after traumatic patellar dislocation in adolescents. J Pediatr Orthop. 2008;28(4):397-402.

15. Walsh SJ, Boyle MJ, Morganti V. Large osteochondral fractures of the lateral femoral condyle in the adolescent: Outcome of bioabsorbable pin fixation. J Bone Jt Surg. 2008;90(7):1473-8.

\section{Nota de contribución:}

Martin Sierra: Autor principal, redactor, trabajo de campo, revisión Bibliográfica.

Marcelo Dupont: Tutor: contribuyó en la redacción de resultados discusión y

conclusiones. Aportó datos descriptivos fundamentales de la técnica quirúrgica.

María Elena Pérez: Asesora científica: aportó pautas de redacción, asesoramiento

sobre las formas metodológicas y contribuyó en las conclusiones.

\section{Nota del Editor:}

El editor responsable del manuscrito es el Dr. Asdrúbal Silveri.

Recibido: 15/09/2020

Aceptado: 08/12/2020 